\title{
Serial ImmuKnow assay in stable kidney transplant recipients
}

\author{
HYUNG HWAN MOON ${ }^{l}$, TAE-SEOK KIM ${ }^{1}$, SANGHOON LEE ${ }^{l}$, SANGHYUN SONG ${ }^{l}$, \\ MILLJAE SHIN ${ }^{1}$, JAE BERM PARK ${ }^{1}$, JONG MAN KIM ${ }^{1}$, HYE RYOUN JANG ${ }^{2}$, WOOSEONG HUH ${ }^{2}$, \\ JAE-WON JOH ${ }^{1}$, SUNG JOO KIM ${ }^{l}$
}

${ }^{1}$ Departments of Surgery, Samsung Medical Center, Sungkyunkwan University School of Medicine, Seoul, Korea

${ }^{2}$ Division of Nephrology, Department of Medicine, Samsung Medical Centre, Sungkyunkwan University School of Medicine, Seoul, Korea

\begin{abstract}
Objectives: The ImmuKnow assay (cylex Inc., Columbia, MD) has been reported to measure the global immune monitoring tool for organ transplantation recipients. We assess immuKnow ATP values in stable kidney transplant patients.

Material and methods: Patients who were kidney transplanted between September 2008 and May 2011 were enrolled in the prospective serial ImmuKnow assay study. The criteria of inclusion were living donor kidney transplantation (KT), no evidence of hepatitis $B$ virus $(H B V)$ and hepatitis $C$ virus $(\mathrm{HCV})$ infection, and PRA less than 50\%. ImmuKnow assay monitoring was performed at one day before operation, post operative weeks 4, 8, 12,16, 20,24, 36 and 52. We excluded patients who had undergone infectious syndrome or rejection episodes during the follow-up period.

Results: Among 71 patients who were enrolled in prospective serial ImmuKnow assay monitoring, 37 patients were proven to stable KT patients during the follow-up period. Two hundred and twenty-four samples from 37 patients were collected. ImmuKnow value and immunosuppression drug level were compared in post operative weeks 4, 8, 12, 16, 20, 24, 36 and 52. The value of ImmuKnow assay was significantly different depending on the length of time after transplant $(p=0.038)$. Interestingly, the pre-transplant ImmuKnow values were lower than those of the immediate post-transplant period.

Conclusions: the ImmuKnow value of stable KT recipients is different according to "time after transplant". Therefore, "time after transplant" should be considered when applying an ImmuKnow assay in clinical practice.
\end{abstract}

Key words: immune monitoring, kidney transplantation, immunosuppression.

(Centr Eur J Immunol 2014; 39 (1): 96-99)

\section{Introduction}

Optimal immunosuppression is an important assignment in the transplant field. To resolve this problem, an appropriate monitoring tool is necessary for the assessment of overall immune function. Recently, the ImmuKnow assay (Cylex, Columbia, MD) has been used to assess overall cell-mediated immune function in transplant patients [1]. The principle of the ImmuKnow assay is to measure the amount of adenosine triphosphate (ATP) produced by CD4 $\mathrm{T}$ cells from whole blood after stimulation with phytohemagglutinin (PHA) [2]. It has been reported that low ATP activity occurs in patients at increased risk for infections, whereas high ATP levels predict rejection [3-5]. However, the intensity of immunosuppression alters depending on the length of time since transplantation and the specific conditions of the recipient. There are few studies concerning the analysis of the serial ImmuKnow assay. In this study, we assessed the ImmuKnow values of stable kidney transplant (KT) recipients according to time after transplant.

\section{Material and methods}

Seventy-one of 335 patients who received KT between September 2008 and May 2011 were enrolled in prospective ImmuKnow assay study. Inclusion criteria were living donor $\mathrm{KT}$ with no evidence of hepatitis B virus (HBV) and hepatitis $\mathrm{C}$ virus (HCV) infection, and PRA less than $50 \%$. ImmuKnow assay monitoring was performed at one day before transplant and post transplant weeks 4, 8, 12, 16, 20, 24, 36 and 52. Whole blood samples collected from these kidney transplant recipients were not used in the management of patients. Among them, patients with no evidence of infection or rejection episode during follow-up were defined as stable. Thirty seven of 71 recipients were 
proven to be stable KT recipients, and their data was used in this study.

All patients received two times basiliximab as induction therapy $\left(20 \mathrm{mg}\right.$, operation day and $4^{\text {th }}$ postoperative day) and high-dose methylprednisolone given $1000 \mathrm{mg}$ on the operation day and then tapered. Maintenance immunosuppression drugs were tacrolimus (FK-506), mycophenolic acid (MPA) and methylprednisone. From first post-transplant day morning, tacrolimus was given. Targets trough levels of tacrolimus were $10-12 \mathrm{ng} / \mathrm{ml}$ until 1 month, $8-10$ $\mathrm{ng} / \mathrm{ml}$ between 1 month and 3 months and 5-8 ng/dl after 3 months. A dose of $540 \mathrm{mg}$ mycophenolic acid was given twice a day from 1 day post-transplant. If neutropenia or gastrointestinal problems occurred, the dose of mycophenolic acid was reduced. Also, we adjusted the dose of mycophenolic acid for severity of viral infection. All patients received prophylactic antimicrobial therapy that included postoperative prophylactic antibiotics, anti-pneumocystis carinii prophylaxis, anti-CMV prophylaxis and anti-fungal prophylaxis. Ceftizoxime was used for postoperative prophylactic antibiotics until postoperative day 2 . Trimethoprim $80 \mathrm{mg}$ and sulfamethoxazole $400 \mathrm{mg}$ were used twice a day for 1 year. For anti-CMV prophylaxis, oral valacyclovir $2000 \mathrm{mg}$ three times a day for 1 month was used. Patients with CMV sero-negative or sero-positive CMV donor received valacylovir $2000 \mathrm{mg}$ three times a days for 3 months. Swallowing $5 \mathrm{ml}$ nystatin four times a day was done in all patients until the $6^{\text {th }}$ postoperative month.

During the first week after transplantation, if the patient had fever or symptoms of infection, bacteria or fungus culture for urine, sputum, drains, wounds and blood were checked. $\mathrm{BK}$ virus replication was screened as planned. Urine cytology or BK virus DNA real-time polymerase chain reaction (PCR) detection was evaluated at POD weeks 1, 5, 9, 16, 24, 36 and 48 up to 1 year. If present, urine BK virus DNA PCR quantitation was tested. CMV antigenaemia had been checked every other day during the first week post transplant. After that, it was checked weekly while hospitalised, then after discharge we checked at every visit (for 4 weeks) until 12 months post transplant. If CMV antigenaemia was over 40 per 400,000 WBCs, IV ganciclovir was given as a preemptive treatment.

\section{Immune function assay}

A total of 224 blood samples were collected from stable KT recipients during the follow-up period. By using ImmuKnow (Cylex, Columbia, MD), the Food and Drug Administration-approved intracellular ATP levels in stimulated CD4 T-lymphocyte were measured.

\section{Statistical analysis}

Statistical analysis was performed using SPSS 18.0 soft ware. Demographic data were recorded as the median with range. The values of the ImmuKnow assay were recorded as the mean with standard deviation. Statisti- cal significances were tested by mixed model in analysis of ImmuKnow assay in BK virus infected patients as time passed. LSD was applied for paired comparison as post-analysis. A $p$ value less than 0.05 was considered statistically significant.

\section{Results}

\section{Demographics}

A total of 37 of 71 patients who received KT between September 2008 and May 2011 were stable KT patients after KT during the follow-up period. Table 1 shows their demographic data. The median age of the patients including 21 males and 16 females was 46 . The median number of hospital days of KT was 14, this is related to our KT in-hospital care program. Median serum creatinine at last follow-up was slightly increased from 1.0 to $1.22 \mathrm{mg} / \mathrm{dl}$. Their mean follow-up time was 30 weeks after KT. A total of 224 blood samples were collected from 37 stable KT recipients. Underlying disease were chronic glomerular nephropathy $(27 \%, 10 / 37)$, hypertensive nephropathy $(21.6 \%, 8 / 37)$, immunoglobulin (Ig) A nephropathy $(16.2 \%, 6 / 37)$, diabetic nephropathy $(8.1 \%, 3 / 37)$, others $(8.1 \%, 3 / 37)$ and unknown $(18.9 \%, 7 / 37)$.

\section{Serial ImmuKnow assay values and FK trough levels of stable KT recipients}

ImmuKnow assay was followed longitudinally during the study period. Figure 1 shows that ImmuKnow value had stabilised and then fixed around 400 ATP $\mathrm{ng} / \mathrm{ml}$ as time went on. There serial ImmuKnow value had significant de-

Table 1. Demographics of stable kidney transplant recipients

\begin{tabular}{lc}
\hline & $N=37$ \\
\hline Male : female & $21: 16$ \\
\hline Donor age & $36(22-61)$ \\
\hline Recipient age & $46(23-64)$ \\
\hline Hospital days & $14(12-23)$ \\
\hline SCR at discharge $(\mathrm{mg} / \mathrm{dl})$ & $1.0(0.64-1.85)$ \\
\hline SCR at last follow-up $(\mathrm{mg} / \mathrm{dl})$ & $1.22(0.71-2.09)$ \\
\hline Median follow-up & 30 weeks \\
\hline Etiology & 10 \\
\hline CGN & 8 \\
\hline hypertensive & 6 \\
\hline IgA & 3 \\
\hline diabetic & 3 \\
\hline others & 7 \\
\hline unknown & \\
\hline
\end{tabular}




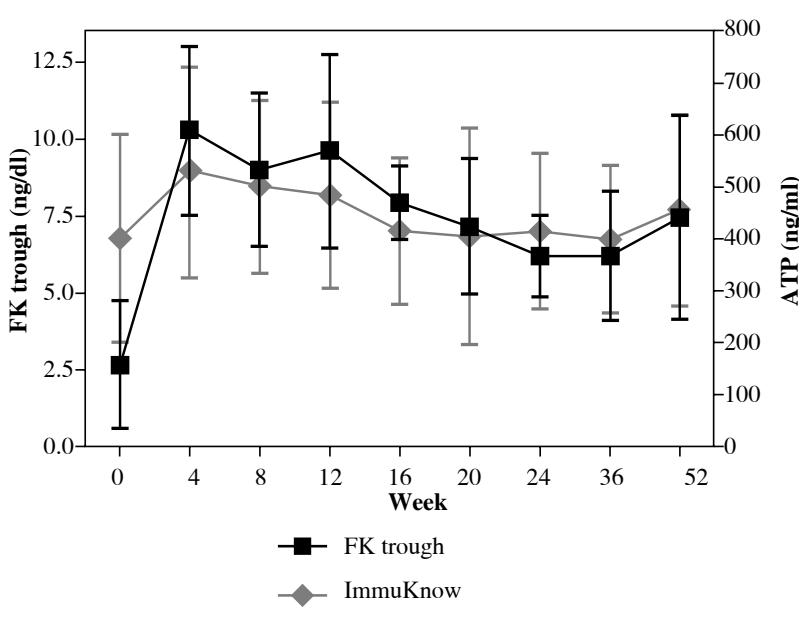

Fig. 1. Serial ImmuKnow assay value and FK trough level of stable recipients after kidney transplantation

pendence on time $(p=0.038)$. Interestingly, the ImmuKnow assay of the pre-transplant state was significantly lower than that seen in the immediate post-transplant period (Table 2).

The significant decrease of FK trough level in post-transplant time is reasonable because we adjusted the dose of tacrolimus according to the target FK trough level according to our protocol. However, the mean ImmuKnow value at the time of pre-transplantation was 401.9 \pm 199 ATP $\mathrm{ng} / \mathrm{ml}$, which was significantly lower than the value of $529 \pm 202 \mathrm{ATP} \mathrm{ng} / \mathrm{ml}$ seen at 4 weeks post-transplant $(\mathrm{P} 4 \mathrm{~W})$ and $502 \pm 165 \mathrm{ATP} \mathrm{ng} / \mathrm{ml}$ at post-transplant 8 weeks (P8W). The mean ImmuKnow value at P4W was highest at $529 \pm 202 \mathrm{ATP} \mathrm{ng} / \mathrm{ml}$ in spite of the highest FK trough level. In addition, although immunosuppression within 12 weeks was stronger than that of the later post-transplant period (after 20 weeks), the ImmuKnow assay at P4W was significantly higher than after 20 weeks

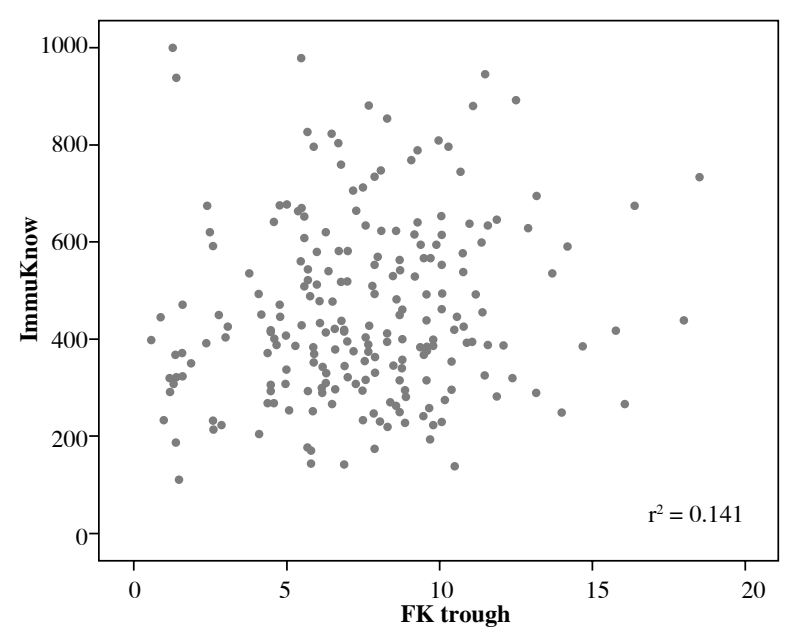

Fig. 2. Correlation between ImmuKnow assay and FK trough level. This graph of correlation between ImmuKnow assay and FK trough level shows mild positive correlation paradoxically

post-transplant (P20W), as shown in Fig. 1. The graph of correlation between ImmuKnow assay and FK trough level shows positive correlation paradoxically (Fig. 2). These results suggest that the FK trough level could not reflect the immunity of transplant patients.

\section{Discussion}

Our study shows that the serial ImmuKnow value changed as time went on. Notably, the ImmuKnow value at the immediate post-transplant period (within P12W) was higher than that of the later post-transplant period (after P20W) and reached around $400 \mathrm{ATP} n g / \mathrm{ml}$ after P20W. In addition, our results show no correlation between ImmuKnow value and FK trough level.

Table 2. Post-analysis* of serial ImmuKnow assay value as time in stable KT recipients

\begin{tabular}{cccccccccccc}
\hline & $\mathbf{0}$ & $\mathbf{4}$ & $\mathbf{8}$ & $\mathbf{1 2}$ & $\mathbf{1 6}$ & $\mathbf{2 0}$ & $\mathbf{2 4}$ & $\mathbf{5 2}$ & 0.768 & 0.594 & 0.863 \\
\hline $\mathbf{0}$ & - & $\mathbf{0 . 0 0 1}$ & $\mathbf{0 . 0 0 9}$ & $\mathbf{0 . 0 8 6}$ & 0.096 & 0.567 & $\mathbf{0 . 0 4 6}$ & $\mathbf{0 . 0 2 9}$ \\
\hline $\mathbf{8}$ & $\mathbf{0 . 0 0 1}$ & - & 0.655 & 0.173 & 0.245 & $\mathbf{0 . 0 2 3}$ & $\mathbf{0 . 0 1 6}$ & $\mathbf{0 . 0 4 2}$ & 0.099 & 0.063 \\
\hline $\mathbf{1 2}$ & $\mathbf{0 . 0 0 9}$ & 0.655 & - & 0.304 & 0.430 & 0.059 & 0.207 & 0.363 & 0.240 \\
\hline $\mathbf{1 6}$ & 0.086 & 0.173 & 0.304 & - & 0.932 & 0.280 & 0.200 & 0.351 & 0.236 \\
\hline $\mathbf{2 0}$ & 0.567 & $\mathbf{0 . 0 2 3}$ & 0.059 & 0.280 & 0.231 & - & 0.786 & 0.981 & 0.767 \\
\hline $\mathbf{2 4}$ & 0.768 & $\mathbf{0 . 0 1 6}$ & $\mathbf{0 . 0 4 2}$ & 0.207 & 0.200 & 0.786 & 0.786 & 0.935 \\
\hline $\mathbf{3 6}$ & 0.594 & $\mathbf{0 . 0 4 6}$ & 0.099 & 0.363 & 0.351 & 0.981 & 0.786 & - \\
\hline $\mathbf{5 2}$ & 0.863 & $\mathbf{0 . 0 2 9}$ & 0.063 & 0.240 & 0.236 & 0.767 & 0.935 & 0.744 \\
\hline
\end{tabular}

*LSD was applied for paired comparison as post-analysis 
A similar result was found in a recent ImmuKnow assay report by He et al. [6] 149 ImmuKnow assays for the 23 event-free patients were made and analysed serially from pre-transplant up to 30 months post-transplant. In that study, the average ImmuKnow values at pre-transplant were lower than those at post-transplant, which were measured 7 days to 30 months later (pre-transplant: $275.6 \pm 19.3 \mathrm{ng} / \mathrm{ml} \mathrm{vs}$. post-transplant $7^{\text {th }}$ day: $\left.354.3 \pm 38.5 \mathrm{mg} / \mathrm{ml}, p=0.06\right)$. In addition, the fluctuations of the ImmuKnow values stabilised after 5 months post-transplant. However, there was no statistical difference among ImmuKnow values.

Serban et al. reported a similar phenomenon in their analysis of serial immune cell function [1]. They found that ATP activity showed moderate to high values in more than $40 \%$ of the samples obtained during the first 3 months post-transplant. Their results showed no correlation between ATP activity and the number of CD4+ T cells or the number of lymphocytes or monocytes. Instead, ATP levels tended to correlate with the number of neutrophils or total white blood cells [1]. The correlation between the ImmuKnow assay and white blood cell count was reported as immune monitoring of cardiac transplant recipients [7]. Those results are unexpected results because ATP, the parameter of immune cellular function, produced by CD4+ T cells in response to phytohemagglutinin [3]. If a high ImmuKnow value during the immediate post-transplant period is associated with increasing white blood cell count as a post-operative change, a relatively high ImmuKnow value immediate post transplant could be interpreted as the natural course of a transplant recipient [8]. Concerning this phenomenon, further study is needed.

It is generally thought that the FK trough level does not reflect the immunity of transplant patients [9]. Our result supports this theory. The graph of correlation between ImmuKnow assay and FK trough level showed a positive correlation paradoxically (Fig. 2). In addition, serial comparison of ImmuKnow and FK trough level also supported this idea.

ImmuKnow value can be classified as follows: low (ATP $\leq 225 \mathrm{ng} / \mathrm{ml}$ ), moderate (ATP 226-524 ng/ml) and high (ATP $\geq 525 \mathrm{ng} / \mathrm{ml}$ ) [10]. A low ImmuKnow value is known to increase the risk of infection and indicate relative risk of infection [11-14]. In the other hand, a high ImmuKnow value suggests the possibility of rejection in many studies $[2,12,15]$. Moreover, the ImmuKnow assay may be used to identify patients with increased risk of short-term mortality [16]. A very low ImmuKnow value may be related to over-immunosuppression and mortality.

However, most studies did not assess longitudinal data after transplantation. In this study, ImmuKnow value data of stable KT suggests as control ImmuKnow data of infection or rejection episodes. The ImmuKnow value of stable KT was relatively high immediately post-transplant and decreased gradually. Therefore, we suggest considering "time after transplant" when applying an ImmuKnow assay in clinical practice.
Authors declare no conflict of interest.

\section{References}

1. Serban G, Whittaker V, Fan J, et al. (2009): Significance of immune cell function monitoring in renal transplantation after Thymoglobulin induction therapy. Hum Immunol 70: 882890 .

2. Kowalski RJ, Post DR, Mannon RB, et al. (2006): Assessing relative risks of infection and rejection: a meta-analysis using an immune function assay. Transplantation 82: 663-668.

3. Sánchez-Velasco P, Rodrigo E, Valero R, et al. (2008): Intracellular ATP concentrations of CD4 cells in kidney transplant patients with and without infection. Clin Transplant 22: 55-60.

4. Gupta S, Mitchell JD, Markham DW, et al. (2008): Utility of the Cylex assay in cardiac transplant recipients. J Heart Lung Transplant 27: 817-822.

5. Bhorade SM, Janata K, Vigneswaran WT, et al. (2008): Cylex ImmuKnow assay levels are lower in lung transplant recipients with infection. J Heart Lung Transplant 27: 990994.

6. He J, Li Y, Zhang H, et al. (2013): Immune function assay (ImmuKnow) as a predictor of allograft rejection and infection in kidney transplantation. Clin Transplant 2013; 27 : E351-358.

7. Israeli M, Ben-Gal T, Yaari V, et al. (2010): Individualized immune monitoring of cardiac transplant recipients by noninvasive longitudinal cellular immunity tests. Transplantation 89: 968-976.

8. Milani TA, Rodrigues L, Chiattone C, Luz JG (2012): Changes in complete blood count in patients with surgically treated facial fractures. J Craniofac Surg 23: e587-591.

9. Schulz-Juergensen S, Burdelski MM, Oellerich M, Brandhorst G (2012): Intracellular ATP production in CD4 T cells as a predictor for infection and allograft rejection in trough level guided pediatric liver transplant recipients. Ther Drug Monit 34: 4-10.

10. Kowalski R, Post D, Schneider MC, et al. (2003): Immune cell function testing: an adjunct to therapeutic drug monitoring in transplant patient management. Clin Transplant 17: 77-88.

11. Moon HH, Kim TS, Roh YN, et al. (2012): Can immune function assay predict infection or recovery? Transplant Proc 2012; 44: 1048-1051.

12. Cadillo-Chavez R, de Echegaray S, Santiago-Delpin EA, et al. (2006): Assessing the risk of infection and rejection in Hispanic renal transplant recipients by means of an adenosine triphosphate release assay. Transplant Proc 38: 918-920.

13. Gautam A, Fischer SA, Yango AF, et al. (2006): Cell mediated immunity (CMI) and post transplant viral infections - role of a functional immune assay to titrate immunosuppression. Int Immunopharmacol 6: 2023-2026.

14. Xue F, Zhang J, Han L, et al. (2010): Immune cell functional assay in monitoring of adult liver transplantation recipients with infection. Transplantation 89: 620-626.

15. Israeli M, Klein T, Sredni B, et al. (2008): ImmuKnow: a new parameter in immune monitoring of pediatric liver transplantation recipients. Liver Transpl 2008; 14: 893-898.

16. Berglund D, Bengtsson M, Biglarnia A, et al. (2011): Screening of mortality in transplant patients using an assay for immune function. Transpl Immunol 24: 246-250. 